\title{
Multiscale Mechanics and Optimization of Gastropod Shells
}

\author{
Mostafa Yourdkhani, Damiano Pasini, Francois Barthelat \\ Department of Mechanical Engineering, McGill University, 817 Sherbrooke Street West, Montreal, QC H3A 2K6, Canada
}

\begin{abstract}
A vast majority of mollusks grow a hard shell for protection. The structure of these shells comprises several levels of hierarchy that increase their strength and their resistance to natural threats. This article focuses on nacreous shells, which are composed of two distinct layers. The outer layer is made of calcite, which is a hard but brittle material, and the inner layer is made of nacre, a tough and ductile material. The inner and outer layers are therefore made of materials with distinct structures and properties. In this article, we demonstrate that this system is optimum to defeat attacks from predators. A two-scale modeling and optimization approach was used. At the macroscale, a two-layer finite element model of a seashell was developed to capture shell geometry. At the microscale, a representative volume element of the microstructure of nacre was used to model the elastic modulus of nacre as well as a multiaxial failure criterion, both expressed as function of microstructural parameters. Experiments were also performed on actual shells of red abalone to validate the results obtained from simulations and gain insight into the way the shell fails under sharp perforation. Both optimization and experimental results revealed that the shell displays optimum performance when two modes of failure coincide within the structure. Finally, guidelines for designing two-layer shells were proposed to improve the performance of engineered protective systems undergoing similar structural and loading conditions.
\end{abstract}

Keywords: seashell, multiscale modeling, representative volume element, failure criterion, nacre, multiscale optimization

Copyright (C) 2011, Jilin University. Published by Elsevier Limited and Science Press. All rights reserved. doi: $10.1016 / \mathrm{S} 1672-6529(11) 60041-3$

\section{Introduction}

Seashells are hard biological structures that are believed to be optimally designed for protection against mechanical threats, and they are now considered as a potential source of inspiration for novel protective devices $^{[1]}$. Many gastropod and bivalve seashells use one or two nacreous shells, which are composed of two distinct materials: an outer layer made of prismatic calcite and an inner iridescent layer made of nacre. The calcitic layer is a hard but brittle material, while on the other hand, nacre is tough and is capable of relatively large inelastic deformation (up to $1-2 \%$ strain) prior to failure $^{[2-4]}$. The combination of a harder layer on the outside with a tougher and more ductile layer on the inside is considered to provide an ideal protective structure ${ }^{[2,4]}$. When the outer surface of a seashell is exposed to a concentrated force in the normal direction, e.g., a predator's bite, the hard ceramic layer can prevent perforation, while the inside layer can absorb mechanical energy that would otherwise be consumed towards more serious damage and catastrophic failure. In case of overloading, the brittle calcite layer may fail due to cracks propagating toward the mollusk soft tissue. Experiments have demonstrated that the tough nacreous layer could delay and eventually arrest those cracks, thereby delaying the complete failure of the shell.

To date, extensive research has been performed on the structure and properties of nacre and calcite but no study has investigated how these two materials operate together in the actual shell. In particular, while there is evidence that nacre is optimized for toughness and energy absorption, little is known about how the shell structure makes the most of its material constituents, i.e. calcite and nacre. The hierarchical structure of seashells as observed in a natural specimen is the result of a multiscale optimization process over millions of years of evolution, from which insights into the design of similar biomimetic protective structures can be gained. In this work, multiscale modeling and optimization are per- 
formed on the structure of a seashell to identify the benefits of its hierarchical structure to resist natural predators.

The article is organized as follow. The macroscale finite element model of the shell is presented first. While the elasticity and failure criterion for the outside calcitic layer are straightforward (the material is linear elastic and brittle), the unique structure and failure mechanism of nacre requires special attention and is treated in the following sections. The macro-microscale model is then presented, followed by an optimization study on the key parameters. Finally, actual perforation experiments on shells are presented to validate the proposed model.

\section{Macroscale modeling of the shell}

At the macroscale, the shell was modeled with finite elements using the commercial package ANSYS (ANSYS, Inc., Canonsburg, PA). Since the actual shape of a shell can be quite complex, in this work a simplified geometry was used while retaining the following features: (i) the shell is composed of two layers; it is curved and has a uniform thickness; (ii) its shape is approximated with a spherical cap; (iii) the edge of the shell is in full, frictionless contact with a rigid substrate (all points of the edge contribute to the load transfer to the ground). Based on these assumptions, an axisymmetric two-layer seashell model was generated (Fig. 1). In this model, the outer and inner layers represent respectively calcite and nacre, with their corresponding material properties.

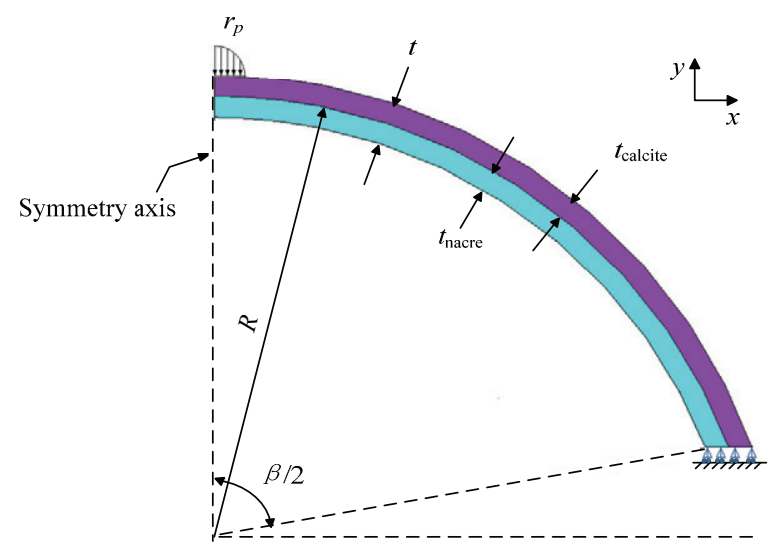

Fig. 1 Two-layer model for the mollusc shell.

ANSYS Plane-42 element was used to mesh the model, which was refined at the region under the area in contact with the external loading to yield the accurate result. In Fig. 1, $R$ is the radius of curvature of the shell (taken at the nacre/calcite interface), $t$ is the thickness of the shell and $\beta$ is the opening angle of the spherical cap. To model a sharp contact load, e.g., from a predator's tooth, a pressure distribution representing Hertzian contact stresses was imposed on a small region at the apex of the shell ${ }^{[5,6]}$. The corresponding applied pressure is

$$
P(r)=\frac{3}{2} P_{m}\left(1-\left(\frac{r}{r_{p}}\right)^{2}\right)^{1 / 2},
$$

where $r_{p}$ is the radius of contact load and $r$ is the distance from an arbitrary point of the distributed load area to the symmetry axis of the shell. $P_{m}$ is the average pressure within the contact region defined as

$$
P_{m}=\frac{P_{\mathrm{Load}}}{\pi r_{p}{ }^{2}}
$$

where $P_{\text {Load }}$ is the total applied load transmitted by the contact. The rim of the shell was assumed to be simply supported and a symmetric boundary condition was imposed on the symmetric axis of the shell.

The outer layer of the shell was modeled as calcite with a Young's modulus of $100 \mathrm{GPa}$ and Poisson's ratio of $0.3^{[7]}$. Since calcite is brittle, its failure was predicted using the maximum principal stress criterion: failure occurred when the largest principal stress at any point of the calcite layer exceeded the tensile strength of calcite $\left(100 \mathrm{MPa}^{[7]}\right)$. The inner layer of the shell is composed of nacre, with modulus and strength highly dependent on its more sophisticated microstructure. Therefore, in order to predict the mechanical properties for nacre, a micromechanical model was developed and is presented in the following section.

\subsection{Microscale modeling of nacre}

Nacre has a well-defined brick and mortar microstructure $^{[4]}$ (Fig. 2a). The flat polygonal "bricks" or "tablets" are separated and bonded by soft organic materials (less than $5 \%$ in volume). In this paper, the inter-lamella distance is termed "interface" and the inter-tablet gap is referred as "junction" (Fig. 2b) ${ }^{[2]}$. The size and the aspect ratio of the tablets largely control the mechanical response of nacre, and interestingly these parameters can vary significantly from one seashell to another ${ }^{[8]}$. For example, in nacre from red abalone, the average tablet diameter and thickness are around $8 \mu \mathrm{m}$ 
and $0.4 \mu \mathrm{m}$, respectively ${ }^{[2]}$, whereas in nacre of Pinctada (oyster) the tablets are around $4 \mu \mathrm{m}$ long and $0.5 \mu \mathrm{m}$ thick $^{[8,9]}$. The thickness of the organic interface in sheet and columnar nacre is much smaller, between 20 and 30 $\mathrm{nm}^{[2,10]}$. In columnar nacre, the tablets are arranged in columns and they are arranged in two distinct regions, namely, the "overlap" and the "core", as defined in Ref. [2]. The "overlap" is the region where two adjacent tablets in two neighbouring layers overlap with each other (Fig. 2b). The remaining part of each tablet, which is not covered in the overlapping region, is referred to as the "core" ${ }^{[2]}$. Overlap region in columnar nacre spans about a third of the area of a tablet. Another type of nacre is sheet nacre, where the arrangement of the tablets from one layer to the next is random. Generally, columnar nacre is found in highly curved shells such as red abalone, whereas sheet nacre occurs more in flatter shells like pearl oyster ${ }^{[11]}$.

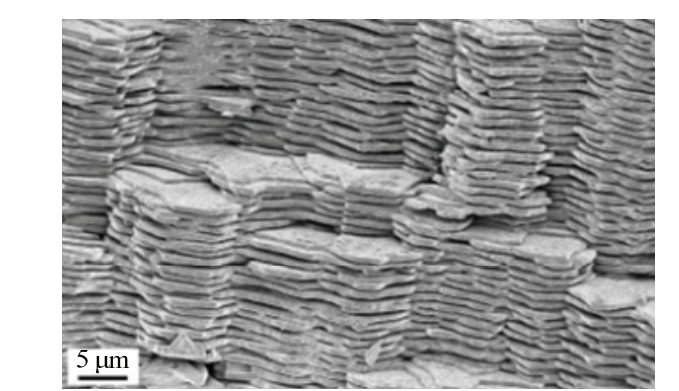

(a)

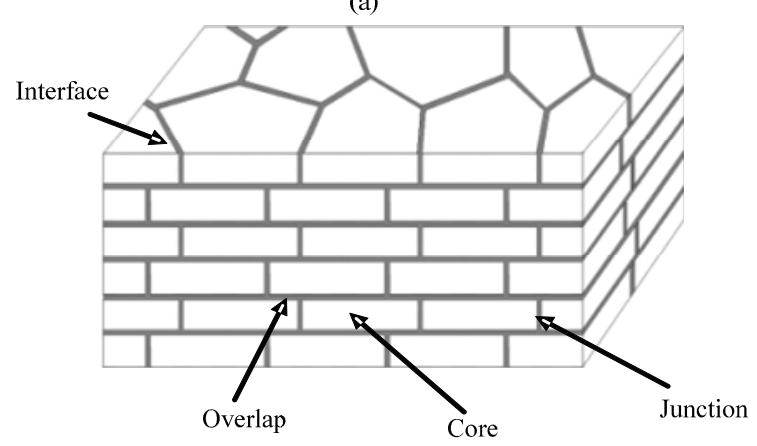

(b)

Fig. 2 Microstructure of nacre: (a) scanning electron micrograph of a fractured surface of nacre; (b) schematic of tablet arrangement in nacre.

In order to capture the structure-properties relationships, an appropriate micromechanics model is required to express the properties of nacre in terms of its microstructural geometry and material properties. Due to its assumed periodic microstructure, the Representative Volume Element (RVE) shown in Fig. 3 is used here to model the mechanical behavior of nacre ${ }^{[9,12]}$.

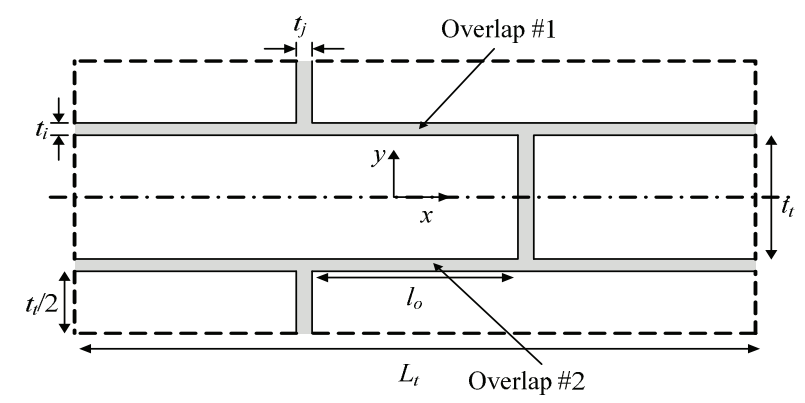

Fig. 3 Schematic of the RVE used to predict the mechanical response of nacre.

In the RVE, $t_{i}, t_{t}, t_{j}, L_{t}$ and $l_{o}$ represent interface thickness, tablet thickness, junction thickness, tablet length and overlap length, respectively. To simplify the analysis while retaining the salient mechanisms of material behavior, the following assumptions are made:

(1) The model is assumed periodic along both directions $x$ and $y$, in terms of geometry, displacements and stress. Thus, the mechanical response of this RVE model is assumed to be representative of the behavior of the whole material ${ }^{[13]}$.

(2) Tablets have uniform thickness with rectangular cross section in the plane of tablets.

(3) Tablets carry only axial stresses while interfaces only transfer shear stresses; thus, the applied stress is carried by shear and tensile elements in series.

(4) Axial and shear stresses are assumed to be uniform across the thickness of the tablets and interface.

(5) Plane stress distribution is considered in the calculations as tablets have finite dimensions and the soft organic interface has the effect of relaxing the constraints applied by the $x-y$ and $x-z$ tablet surfaces, which make those surfaces free surfaces. As a result, the Poisson's ratio effect is neglected.

Note that similar assumptions were made in a previous work on a similar model ${ }^{[9]}$.

\subsubsection{In-plane elastic modulus}

A mathematical model for the in-plane elastic modulus of nacre was first proposed by Kotha et al. ${ }^{[9]}$. The solution was obtained for fully overlapped nacre, and by neglecting the effect of junctions between two adjacent tablets of the same layer. Since the overlap region transfers axial stress between the tablets ${ }^{[14]}$, its length plays an important role on the total stress transfer as well as on the elastic modulus. Here, Kotha's 
expression is revisited to include arbitrary overlap lengths and the contributions of the junctions. To make the mechanical properties of nacre independent on its microscale dimensions, nondimensional parameters were defined by normalizing the microstructural dimensions by the tablet length as

$$
\bar{t}_{t}=\frac{t_{t}}{L_{t}}, \bar{t}_{i}=\frac{t_{i}}{L_{t}}, \bar{t}_{j}=\frac{t_{j}}{L_{t}}, \bar{l}_{o}=\frac{l_{o}}{L_{t}} .
$$

The resulting in-plane elastic modulus of nacre is found to be

$$
E_{\text {In-plane }}=\frac{\bar{t}_{t}}{\overline{t_{t}}+\bar{t}_{i}} \frac{1}{\left(\frac{1}{E_{t}}+\frac{\bar{t}_{j}}{E_{i}} \frac{K}{1+K}\right)},
$$

where $K$ is

$$
K=\left(\frac{\alpha \overline{t_{t} t_{i}}}{2 \bar{t}_{j}} \frac{E_{i}}{G_{i}}\right)\left[\frac{1+\cosh \left(\alpha \bar{l}_{o}\right)}{\sinh \left(\alpha \bar{l}_{o}\right)}+\frac{1+\cosh \left(\alpha\left(1-\bar{l}_{o}\right)\right)}{\sinh \left(\alpha\left(1-\bar{l}_{o}\right)\right)}\right] \text { (5) }
$$

and

$$
\alpha=\sqrt{\frac{4 G_{i}}{\overline{t_{t} t_{i}} E_{t}}}
$$

where $E_{i}$ is the elastic modulus of the interface; $E_{t}$ is the elastic modulus of the tablet; and $G_{i}$ is the shear modulus of the interface.

This expression shows how nacre microstructure controls its elastic modulus. For example, thinner interface or longer overlap has the effect of increasing the in-plane elastic modulus.

Out-of-plane elastic and shear moduli were formulated using the Reuss composite model ${ }^{[15]}$ and the in-plane shear modulus was formulated by assuming isotropic property in the plane of the tablets, i.e.

$$
G_{\text {In-plane }}=\frac{E_{\text {In-plane }}}{2(1+v)}
$$

where $v$ is the corresponding Poisson's ratio with a value of $0.2^{[16]}$, a usual value for ceramic materials ${ }^{[17]}$.

\subsubsection{Failure criterion}

The choice of an appropriate failure criterion is required in order to predict the failure of nacre under multiaxial stresses. Nacre displays several types of failure modes, which cannot be properly captured by the failure criteria typically used for engineering composite materials. For this reason, we formulated a new micromechanics-based multiaxial failure criterion for nacre. Considering the RVE shown in Fig. 3, several types of failure are possible:

i) In tension along the tablets, the interface in the overlap region may yield in shear, thereby leading to tablets sliding on one another. This type of failure has been observed experimentally ${ }^{[14]}$.

ii) In tension along the tablets, the stresses in the tablet may reach the tensile strength of the mineral, which leads to a brittle failure.

iii) When subjected to shear stresses, the interfaces can yield, leading to a shear failure.

iv) In tension across the tablets, the interfaces easily fail and nacre cleaves in a brittle fashion.

v) In compression, the material is much stronger ${ }^{[15]}$. When the state of stress in the seashell is examined, tensile and compressive stresses exist. Failure by tensile stress occurs first and for this reason, compressive failure was not considered.

Experiments on nacre demonstrated that the prominent failure mode of nacre in tension along the tablets is the first failure mode (tablet sliding). However, when nacre is loaded in shear or tension across the tablet, the failure of the organic interface controls nacre failure (mode iii and iv). The material is assumed to have failed when any point of the RVE has failed. This reasoning was first employed for predicting the failure of composite materials subject to macroscale stresses by developing a micromechanical model that captures all possible failure mechanisms at the microscale ${ }^{[18,19]}$. Although conservative, this approach showed to be appropriate to predict the failure of the entire structure.

The basic idea to derive the failure criterion is to model the yielded interface with a series of parallel springs that can be stretched along and across the tablets to the extent defined by the finite strength of the organic material. When nacre is loaded in tension, the stress parallel to the plane of the tablets, $\sigma_{x x}$, is transferred from one tablet to another through the organic interface in the form of shear stresses. These shear stresses include the shear stress developed at the overlap $\left(\tau_{\text {tension }}\right)$ and the yield stress of the junction exerted on the tablets $\left(\sigma_{i}{ }^{y}\right)$. The equilibrium equation can be written as

$$
\sigma_{x x}\left(t_{t}+t_{i}\right)=\tau_{\text {tension }}\left(l_{o}\right)+\sigma_{i}^{y} t_{t} .
$$


Rearranging Eq. (8) gives

$$
\tau_{\text {tension }}=\frac{\sigma_{x x}\left(t_{t}+t_{i}\right)-\sigma_{i}^{y} t_{t}}{l_{o}} .
$$

By superposition, the total shear stress at the interface of the overlap region $\left(\tau_{i}\right)$ is given by adding the external shear stress $\left(\tau_{x y}\right)$ to the shear stress developed by the axial stress as

$$
\tau_{i}=\tau_{\text {tension }}+\tau_{x y} .
$$

Furthermore, transverse stress $\left(\sigma_{i}\right)$ can be developed at the interface as a result of direct transfer of the normal stress across the tablet layers $\left(\sigma_{y y}\right)$ as

$$
\sigma_{i}=\sigma_{y y} .
$$

The resultant force generated by the transverse and shear stresses at the organic interface of the overlap can be obtained by multiplying it by the largest area of the tablet $\left(A_{\text {Tablet }}\right)$ as

$$
\left(\tau_{i} A_{\text {Tablet }}\right)^{2}+\left(\sigma_{i} A_{\text {Tablet }}\right)^{2}=F_{y}{ }^{2},
$$

where $F_{y}$ is the load bearing capacity of the organic spring at failure. Dividing Eq. (12) by $A^{2}$ Tablet gives

$$
\tau_{i}^{2}+\sigma_{i}^{2}=\frac{F_{y}^{2}}{A_{\text {Tablet }}^{2}}=\left(\sigma_{i}^{y}\right)^{2} .
$$

Substituting Eqs. (9), (10) and (11) into Eq. (13) and normalizing the latter with respect to the tablet length, we obtain

$$
{\sigma_{y y}}^{2}+\left(\frac{\sigma_{x x}\left(\bar{t}_{t}+\bar{t}_{i}\right)-\sigma_{i}{ }^{y} \bar{t}_{t}}{\bar{l}_{o}}+\left|\tau_{x y}\right|\right)^{2}=\left(\sigma_{i}^{y}\right)^{2},
$$

which provides a failure criterion for nacre. It is worth mentioning that regardless of the sign of the external shear stress, the two shear stress components, i.e., the external shear stress and the shear stress induced on the interface by the axial tensile stress, are in the same direction in one of the two overlaps (Fig. 3). Since there is always a critical overlap susceptible to failure, the absolute sign is used in Eq. (14) for the external shear stress.

To predict nacre failure in the seashell finite element model, the failure criterion derived in Eq. (14) for multi-axial states of stress was used. Compressive stresses in nacreous layer were considered for few typical cases to assess whether nacre fails in tension or compression; it was found that nacre likely fails in tension rather than in compression because its compressive strength is significantly higher than its tensile strength ${ }^{[20]}$. Therefore, the effect of compressive stresses was not considered further.

\subsection{Failure prediction for the entire shell}

The overall approach to predict the failure of the shell using the finite element model described in the preceding section was as follow. A load of $1 \mathrm{kN}$ was applied at the apex of the shell through the spherical contact pressure distribution described by Eq. (1). The finite element model returned the corresponding stresses in the calcitic and nacreous layers. These stresses were then compared to the failure criteria for the calcitic (maximum tensile stress) and nacreous layers. Using the failure criteria for nacre and calcite, a "failure factor" $(N)$ was calculated for both layers to detect the critical point of the shell, which is prone to fail first. For the calcitic layer, $N$ is defined as

$$
N_{\text {Calcite }}=\max \left\{\begin{array}{ll}
\frac{\max \left\{\sigma_{1}, \sigma_{2}, \sigma_{3}\right\}}{S_{u t_{\text {Calcite }}}}: & \operatorname{assuming} \sigma_{1}>0 \\
\frac{\min \left\{\sigma_{1}, \sigma_{2}, \sigma_{3}\right\}}{-S_{u c_{\text {Calcite }}}}: & \operatorname{assuming} \sigma_{3}<0
\end{array},\right.
$$

where $\sigma_{1}, \sigma_{2}$ and $\sigma_{3}$ are the principal stress components and $S_{u t_{\text {Calcite }}}$ and $S_{u c_{\text {Calcite }}}$ are the ultimate tensile and compressive stresses of calcite, respectively. In Eq. (15), the effects of the tensile and compressive stresses were considered to predict rupture in calcite. Similarly, $N$ for the nacreous layer is defined as

$$
N_{\text {Nacre }}=\frac{\text { Nacre Equivalent Stress }}{\text { Transvere Strength of Nacre }}=\frac{\sigma_{\text {Nacre-eq }}}{S_{\text {Nacre-transverse }}}
$$

where $\sigma_{\text {Nacre-eq }}$ is the equivalent stress for nacre using Eq. (14)

$$
\sigma_{\text {Nacre-eq }}=\sqrt{\sigma_{y y}{ }^{2}+\left(\frac{\sigma_{x x}\left(\bar{t}_{t}+\bar{t}_{i}\right)-\sigma_{i}{ }^{y} \bar{t}_{t}}{\bar{l}_{o}}+\left|\tau_{x y}\right|\right)^{2}} .
$$

For a given load, the stresses and consequently the failure factor for all points of the structure are calculated. The point with the highest $N$ is prone to failure prior to other points. 


$$
N_{F}=\max \left(N_{\text {Nacre }} \text { and } N_{\text {Calcite }}\right) \text {. }
$$

$N_{F}$ depends on the amount of the load exerted on the shell. When $N_{F}$ is less than unity, the point for which $N_{F}$ is calculated is safe; on the other hand, $N_{F}=1$ means that failure occurs within the structure. The maximum load that the structure can support is the amount of load for which $N_{F}$ is equal to unity. Since the system is linear, the maximum load is calculated as

$$
P_{\max }=\frac{P_{\text {Initial }}}{N_{F}},
$$

where $P_{\max }$ is the maximum load that the shell can carry and $P_{\text {initial }}$ is the assumed load of $1 \mathrm{kN}$ used to solve the problem and compute $N_{F}$. With this approach, the ultimate load that the shell can carry is obtained and the location of the critical point is also identified. Based on this approach, failure can occur at different locations on the shell depending on the geometrical parameters of the shell, i.e., shell thickness, the average radius of curvature of the shell and the ratio of calcite to the shell thickness $^{[21]}$. The thickness of the shell and the amount of calcite in the structure were found to be the key parameters in locating the failure. As a result, the followings were identified to govern the failure modes of the shell:

Failure due to contact stresses: In this case, failure from Hertzian contact stresses in the calcite layer is prominent. The configuration near the contact point is similar to a sphere in contact with a half space. According to the contact stress field, the maximum principle stress occurs on the shell surface, right at the edge of the contact area. In practice, this stress generates Hertzian conical crack $^{[6]}$. This type of failure was predicted for shells with a thick calcitic layer (Fig. 4a).

Failure at the interface of two layers (nacreous side): For a thick shell with a thin calcite layer, the model predicted failure at the calcite/nacre interface on the nacreous side. This is the case where the calcite/nacre interface was sufficiently close to the surface so that the nacreous layer could "sense" the contact stresses. Since nacre has lower strength than calcite, its microstructure is prone to failure although the stresses on the points of the two sides of the interface are the same (Fig. 4b).

Failure due to flexural stresses: Flexural stresses are generated by the bending of the shell. Flexural fail- ure was predicted at the bottom of the nacreous layer (inner surface of shell) (Fig. 4c), and was observed when the overall thickness of the shell was decreased.

Which one(s) of these failure modes prevails is determined by the overall shell geometry and by the microstructure of nacre. In the next section, an optimization study of the seashell structure that captures the effect of these failure modes is presented.

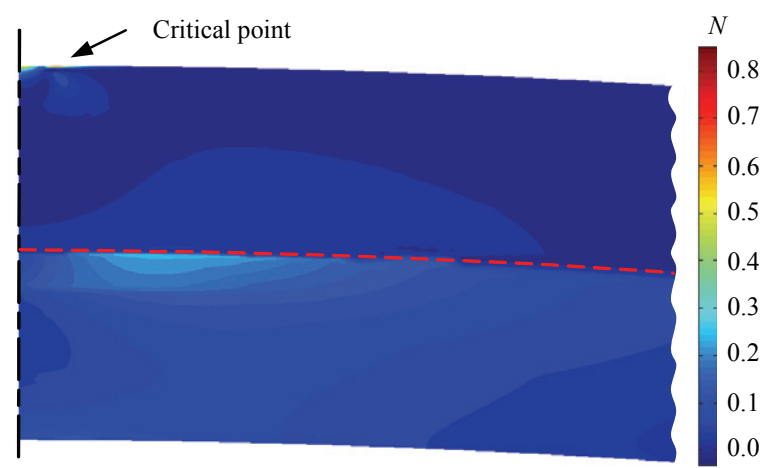

(a)

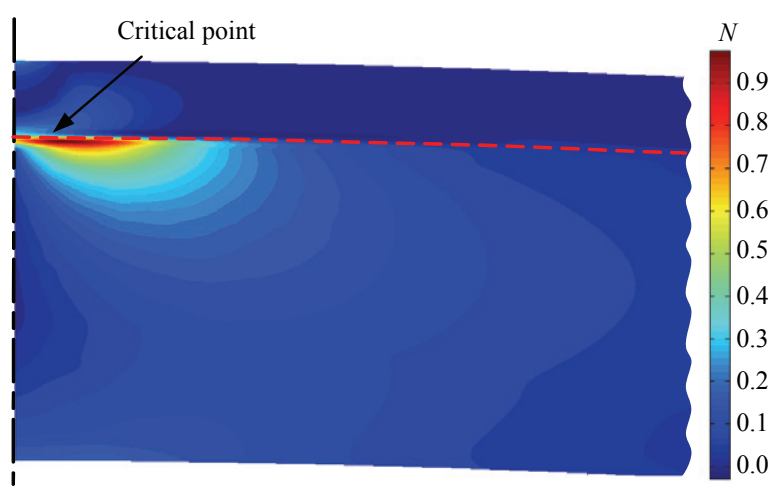

(b)

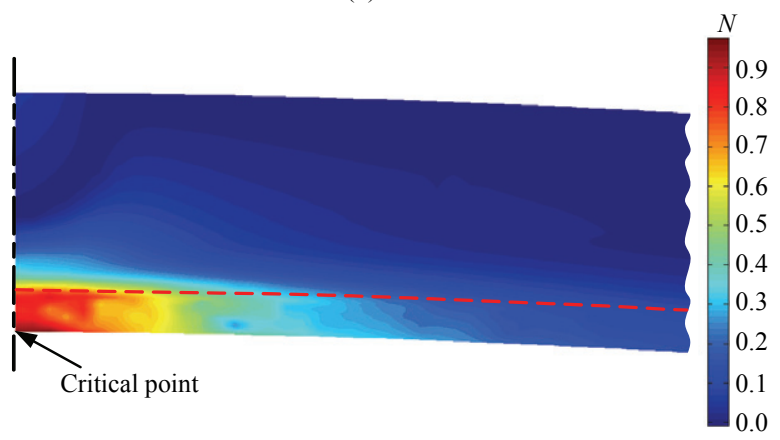

(c)

Fig. 4 Contour plots of failure factor $(N)$ to determine the failure point. (a) failure at the calcite layer due to contact stresses; (b) failure initiated at the interface of two layers in nacreous side; (c) failure at the inner surface of the shell due to flexural stress.

\section{Multiscale optimization}

Structural optimization is used in this section to investigate how the overall structure of the shell and the 
microstructure of nacre are optimized to resist perforation. Based on the finite element model explained in the preceding section, the geometry of the shell at the macroscale is described by the parameters $R, t, \beta$ and $\alpha_{c}$. In addition, the radius of the contact area is another variable that expresses the load distribution. Since the load carrying capacity of the shell depends on the load contact area, both the thickness and the radius of curvature were normalized with respect to the contact radius

$$
\bar{R}=\frac{R}{r_{p}}, \bar{t}=\frac{t}{r_{p}}
$$

Consequently, the results of the modeling and multiscale optimization can be scaled for any structure with similar geometry and loading conditions. In addition to the structural variables at the macroscale, three normalized microscale variables, i.e., $\bar{t}_{t}, \bar{t}_{i}$, and $\bar{l}_{o}$ control the mechanical behavior of nacre. It was assumed that the junction has the same thickness as the interface.

The optimization problem was formulated as follows

$$
\operatorname{maximize} \frac{P_{\mathrm{Load}}}{r_{p}^{2}}=f\left(\bar{R}, \bar{t}, \alpha_{c}, \bar{t}_{t}, \bar{t}_{i}, \bar{l}_{o}\right)
$$

subject to the constraints: $\left\{\begin{array}{l}10<\bar{R}<800, \\ 1<\bar{t}<40, \\ 0.05<\alpha_{c}<0.95 \\ 0.02<\bar{t}_{t}<2, \\ 0.001<\overline{t_{i}}<0.5, \\ 0.1<\bar{l}_{o}<0.5 .\end{array}\right.$

The constraints restricted the parameters to feasible regions of the design space where the variables have practical and realistic values.

\subsection{Optimization with respect to all multiscale vari- ables}

In the multiscale optimization problem, the parameters controlling the microstructure of nacre and the geometry of the shell are design variables, and the objective function to be maximized is the overall strength of the shell. The mechanical properties of the constituents are parameters kept constant. As a result, $\left(\bar{R}, \bar{t}, \alpha_{c}\right)$ and $\left(\bar{t}_{t}, \bar{t}_{i}, \bar{l}_{o}\right)$ are respectively the macroscale and microscale variables of the optimization problem. The shell opening angle was fixed at $140^{\circ}$, similar to that of an actual shell of red abalone.

The Nelder-Mead (NM) simplex search method ${ }^{[22]}$ was applied to optimize the shell with respect to all six multiscale parameters. Since the NM algorithm is prone to find only local optima, the NM optimization process was coupled to a probabilistic restart for the search of the global optimum. Thus, NM was started at 100 randomly picked initial simplexes within the design space to increase the chance of converging to the global optimum. As expected, the algorithm converged to various points within the design space due to the presence of multiple local optima in the objective function. The best six results, which remarkably exhibit higher loads than the other results, were selected and shown in Table 1. As seen in Table 1, the results do not converge to a single point; however, all parameters fall into a small range compared to the boundaries assigned to each variable (Eq. (21)). The optimum shell parameters lie in a narrow range of structural and material variables rather than converging to a single point, as it is found in nature where seashells of the same type exist in various geometries. By examining the results of the microscale variables, it is observed that the optimum tablet and interface dimensions reached the lower boundaries. The reason is that by making the tablet and interface thinner, the modulus and strength of nacre increase. In practice, these dimensions cannot be too small because it could lead to the tablet failure (which is determined by the high stress transfer within the tablets). This phenomenon is also observed in nature where the tablet aspect ratio can reach a limit where the tablets, rather than the organic interface, fracture ${ }^{[8]}$. The variation in the overlap size is also in agreement with existing information about columnar and sheet nacre ${ }^{[2,10]}$.

\begin{tabular}{|c|c|c|c|c|c|c|c|}
\hline & \multicolumn{3}{|c|}{ Shell geometry } & \multicolumn{3}{|c|}{ Microstructure } & \multirow{2}{*}{$\begin{array}{c}P_{\max } /\left(r_{p}\right)^{2} \\
(\mathrm{GPa})\end{array}$} \\
\hline & $R^{*}$ & $t^{*}$ & $\alpha_{c}$ & $t_{t}^{*}$ & $t_{i}^{*}$ & $l_{o}^{*}$ & \\
\hline Run \#1 & 366 & 8.5 & 0.63 & 0.02 & 0.001 & 0.44 & 7.354 \\
\hline Run \#2 & 333 & 8.5 & 0.62 & 0.02 & 0.001 & 0.34 & 7.298 \\
\hline Run \#3 & 428 & 9.4 & 0.66 & 0.02 & 0.003 & 0.20 & 7.207 \\
\hline Run \#4 & 407 & 11.0 & 0.78 & 0.02 & 0.003 & 0.36 & 6.143 \\
\hline Run \#5 & 412 & 15.8 & 0.34 & 0.05 & 0.012 & 0.40 & 6.097 \\
\hline Run \#6 & 391 & 17.2 & 0.37 & 0.07 & 0.038 & 0.44 & 5.934 \\
\hline
\end{tabular}

Table 1 Results of multiscale optimization (six variables)

In addition to monitoring the overall strength of the shell for various combinations of the macroscale and 
microscale parameters, the failure mode was identified for each case. Interestingly, the optimum combinations in terms of strength coincide with the transition between two of the failure modes identified above: Hertzian contact failure in calcite layer and failure in nacreous layer initiated at the interface of two layers. The concept of coincidence of different failure modes in a structure was first explained by Weaver and Ashby ${ }^{[23]}$, who showed that a column is optimally designed when all possible modes of failure occur concurrently within the structure. The same scenario was observed here. The seashell makes the most of its constituent materials when the modes of failures occur all simultaneously. As a result, the failure damage of the strongest seashell, capable of maximizing the load resistance, is distributed evenly in two layers rather than being concentrated in a specific location.

\subsection{Optimization with respect to $\bar{t}$ and $\alpha_{c}$}

In the preceding section, the results of the optimization study with respect to all multiscale parameters were reported. However, by performing a sensitivity analysis on all design variables it was found that among all six multiscale parameters, two macroscale parameters, i.e., normalized shell thickness $(\bar{t})$ and the calcite to shell thickness ratio $\left(\alpha_{c}\right)$, have significant influence on the load resistance. To capture this influence, the optimization problem is simplified to $\bar{t}-\alpha_{c}$ design space at this point while all other variables are kept constant. A dimensionless radius of curvature, $\bar{R}$, of 200 was assumed to simulate a sharp load on the shell. Microstructural variables of nacre were also selected according to data available for the nacre of red abalone shell, i.e., $\bar{t}_{t}, \bar{t}_{i}$ and $\bar{l}_{o}$ equal to $0.05,0.0037$ and 0.2 , respectively ${ }^{[2]}$. Similarly to the previous section, the NM simplex search method was used and initiated at 100 initial simplexes to find the optimum shell structure. The results are shown in Fig. 5a, where the red solid circles represent the converged results of each initial guess. The blue solid triangles, on the other hand, represent transition between various failure modes.

To visualize the effect that each parameter can have on the load carrying capacity of the shell and validate the optimization results, the maximum load for any possible combinations of the variables $\bar{t}$ and $\alpha_{c}$ was achieved and illustrated in Fig. 5b. The results show that the highest load to failure is achieved for combinations of $\bar{t}$ and $\alpha_{c}$ lying at the crest of the curved ridge shown in Fig. $5 \mathrm{~b}$. This ridge corresponds to the transition failure boundaries illustrated in Fig. 5a. The ridge in the design space map, therefore, matches the transition between failure modes in calcite layer and failure in nacreous layer. This map helps to gain insight into the values of $\bar{t}$ and $\alpha_{c}$ for which the seashell structure maximizes its failure resistance, which occurs when two failure modes appear concurrently.

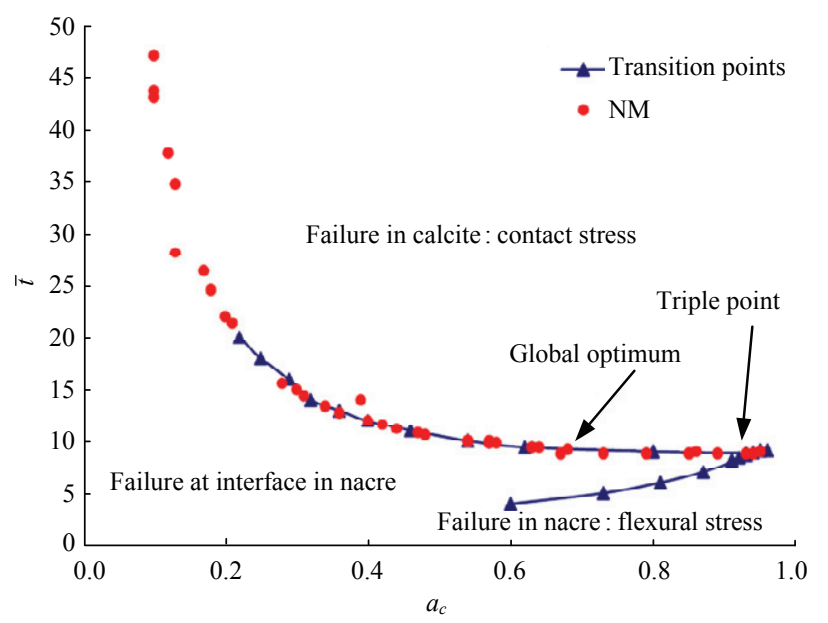

(a)

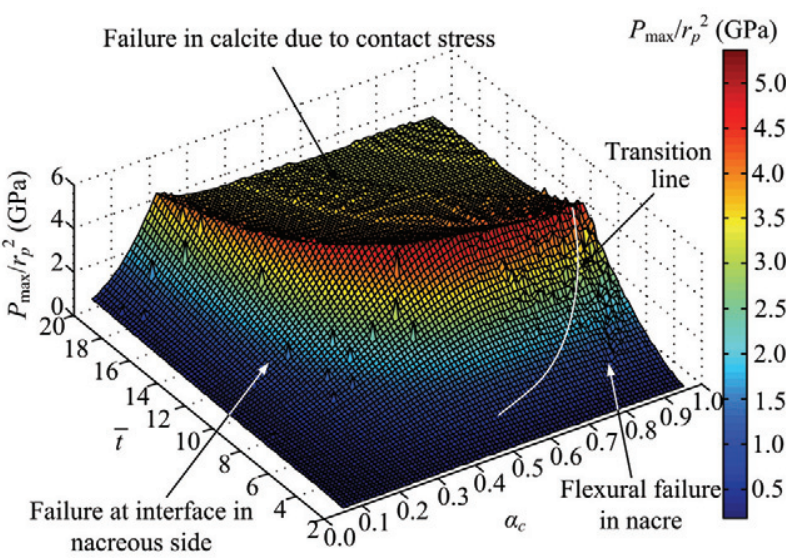

Fig. 5 (a) Optimization results for NM algorithm initiated from different points within the design space. Transition between three failure modes was also determined to understand the relation between failure modes and the converged results; (b) visual map of design space for $\bar{t}$ and $\alpha_{c}$.

\section{Perforation experiments on actual seashells}

In order to validate the findings from modeling and optimization, actual perforation experiments were performed on whole red abalone shells. Two intact shells of red abalone were purchased from a shell shop (Specimen 
shells, Halifax, NS) and stored in water to maintain hydration. For the experiments, the shells were taken out of the water and installed on a custom made fixture (Fig. 6) in a mechanical loading machine (MTS Systems Corporation, Eden Prairie, MN). The shells were punctured at their apex using an indenter consisting of $1 \mathrm{~mm}$ diameter tungsten ball mounted on a short steel cylinder, and attached to the load cell of the loading machine. The inner surface of the shell underneath the indentation site was imaged through a hole in the base plate with two CCD cameras. Flexible links carrying lights at their tips were employed to illuminate the area inside the shell (Fig. 6). VIC3D digital image correlation system (Correlated Solutions, Columbia, SC) was then utilized to determine the three dimensional displacement field of the inner surface of the shell from the stereo imaging information from the two cameras. The surface inside the shell was covered with a very thin layer of white mat paint and black speckles were sprayed on the white background to generate a random pattern. During the experiments, the crosshead of the loading stage was moved down with a rate of $0.5 \mathrm{~mm} \cdot \mathrm{s}^{-1}$. VIC3D system was set up to save two photos per second of the desired surface.

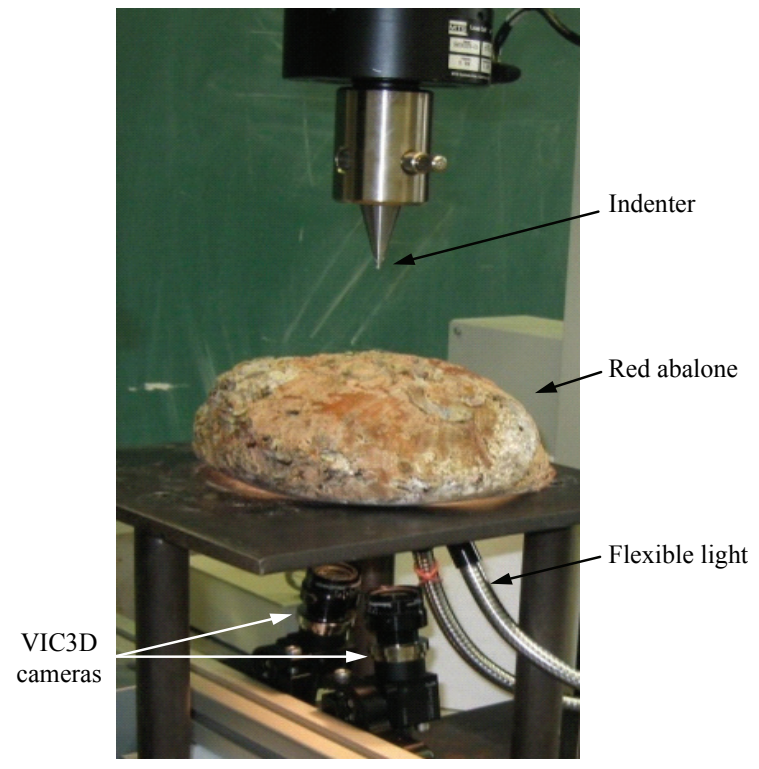

Fig. 6 Experimental setup for perforation test.

Load versus crosshead displacement curves of the two experiments are shown together in Fig. 7a. From the experimental results, it was observed that the failure behavior of the two samples followed identical patterns. The load-displacement curve of the first experiment was chosen to explain the mechanism under which the seashell failed under perforation (Fig. 7b). From the origin to point $\mathrm{A}$, the indenter penetrated into the calcite material. The reason for this is that calcite in the red abalone shell appeared to contain voids, thus their collapse under the indenter led to local crushing and perforation. At point $\mathrm{A}$, the shoulder of the indenter, which comprised the tungsten carbide ball, touched the surface of the calcite layer besides the ball and contributed to the load transfer. After this point, the load had to be increased to penetrate into the calcite material. However, with the same amount of load the shell started to bend and the flexural stresses at the inner surface of the shell rose until point $\mathrm{F}$, where a tiny crack was detected in nacre. The load drop at point B was associated with a small piece of the rim of the shell breaking. The flexural crack at point $\mathrm{D}$ is shown in Fig. 8a.

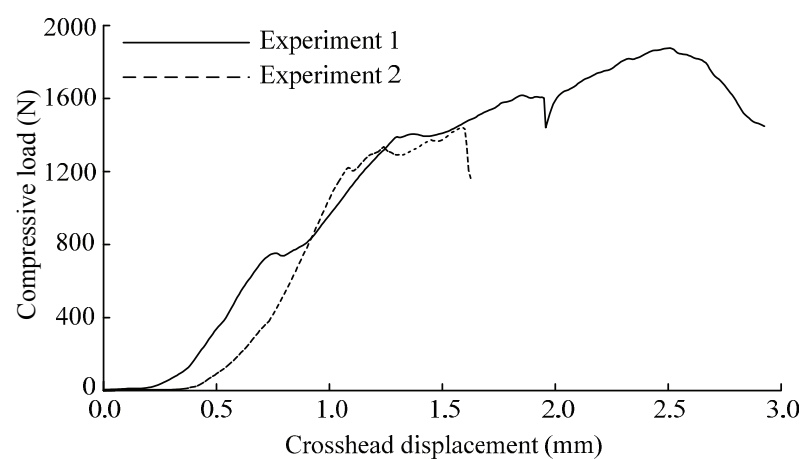

(a)

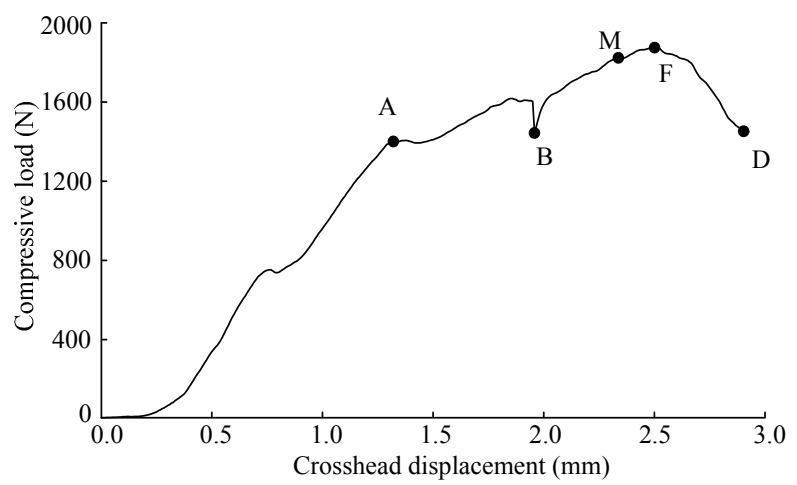

(b)

Fig. 7 Load-crosshead displacement curve for (a) two experiments; (b) experiment 1 only, showing different stages: at point A, indenter touched the surface of the shell; a piece of the rim of the shell broke at point B; the image of inner surface of the shell at point $\mathrm{M}$ was used in digital image correlation to calculate the principal strain; failure of the shell occurred at point $F$.

The test was continued after point $\mathrm{D}$ while acquiring photos from the inner surface of the shell to monitor the failure behavior. The flexural crack at the inner surface of the shell opened until the point where the 
indenter pierced more deeply into the calcite material. At this instant, the influence of Hertzian contact stresses reached the interface of two layers and the nacreous material felt the high Hetzian contact stresses; therefore, the conical crack propagated into the nacreous layer and moved toward the inner surface of the shell where it finally merged with the flexural crack and then, formed a circular crack on the surface (Fig. 8b). As a result, a conical plug detached from the shell (Fig. 8c). The shell was recovered after the experiment and it was cut in half through the center of the conical hole using a diamond saw. The two sections of the shell passing through the hole are shown in Fig. 8d. Several crack paths were observed in the nacreous layer. The main crack determined the conical hole with a large angle with respect to the axis perpendicular to the inner surface. As seen in Fig. 8d, some parts of material were pulled out of the nacreous layer and a crack was visible around those regions. In addition, at the interface of two layers, a delamination crack was detected.

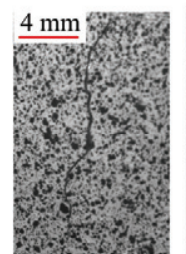

(a)
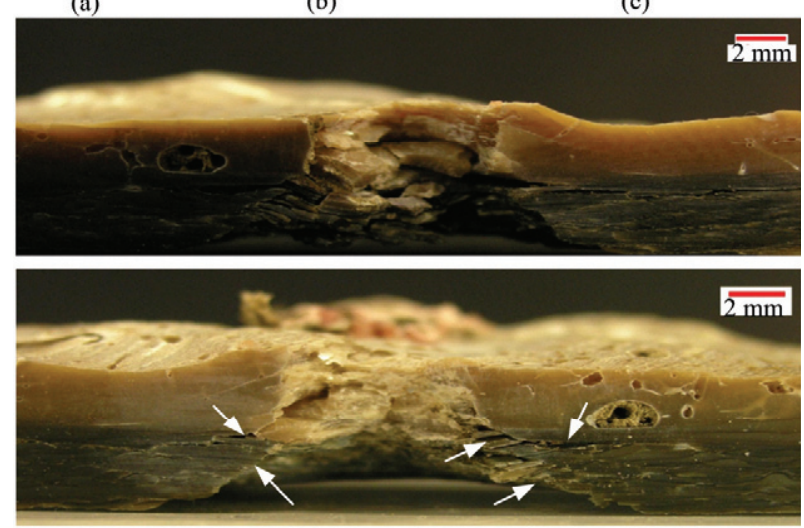

(d)

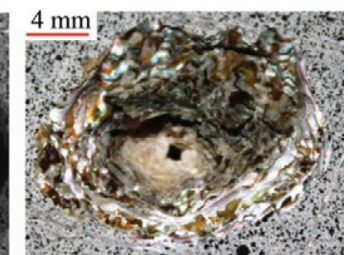

$\overline{\mathrm{mm}}$
Fig. 8 (a) Flexural crack initiated on the inner surface of the shell; (b) circular crack generated after the flexural and conical crack merged together; (c) conical hole formed inside the shell at the end of the experiment; (d) two cross sections of the shell after being cut with diamond saw through the punctured hole. Arrows show the crack propagation path.

The experimental observations suggest that the crack in nacre preferably propagated through the organic interface between the tablets. Crack branching is also observed, which is an identified way to increase the overall strength $^{[3]}$. In crack branching, the crack is distributed into two or more branches, which decelerates the failure by spreading the crack along different directions and reducing the stress intensity at the tip of the crack. Interestingly, in both experiments the three modes of failure were observed, which suggest that they occur simultaneously or almost simultaneously. This observation is consistent with the finding of the multiscale optimization study.

Using three-dimensional digital image correlation, the first principal strain was computed for the area on the inner surface of the shell and underneath the location of the indenter. Fig. 9 depicts the principal strain for point $\mathrm{M}$ of Fig. $7 \mathrm{~b}$ before the point where flexural crack was detected. A high strain region is observed around the line where the flexural crack will be initiated afterward. The maximum tensile strains reached $1.2 \%$, which is consistent with the maximum strain nacre can withstand in tension ${ }^{[16]}$.

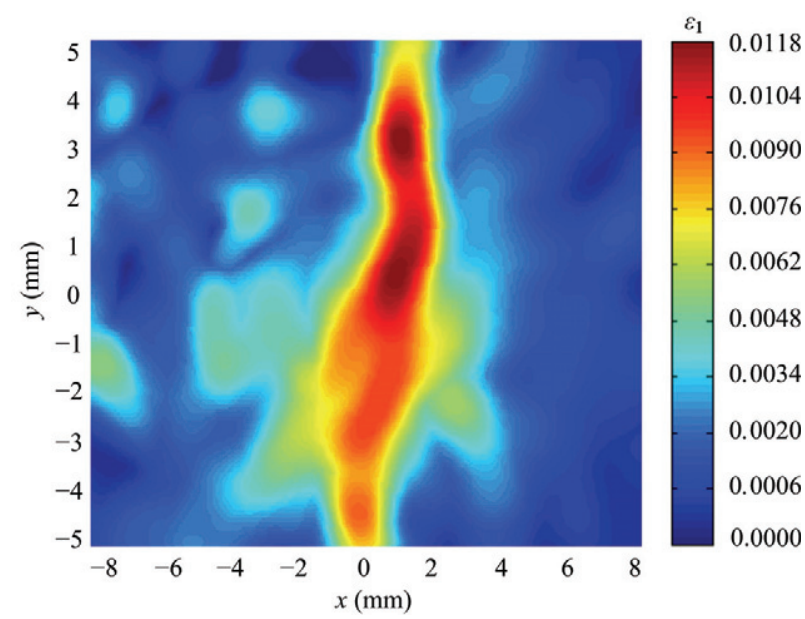

Fig. 9 First principal strain on the nacreous surface underneath the indenter, just before the initiation of flexural cracks.

To calculate the load from the numerical modeling, the radius of contact area should be estimated from the experiments. Tungsten carbide ball had a radius of $0.5 \mathrm{~mm}$ but as it was observed during the experiments, the shoulder of the tip of the indenter, whose radius was $0.75 \mathrm{~mm}$, also contributed to the load transfer. Therefore, the contact radius in the modeling was assumed to be $0.75 \mathrm{~mm}$ to represent the contact after the indenter touched the surface of the shell. Although the contact surface was not in a spherical profile when the indenter touched the surface of the shell, the whole contact was assumed to occur through a sphere. 
Table 2 Comparison of maximum load of modeling and experiments

\begin{tabular}{lccccc}
\hline & \multirow{2}{*}{$R(\mathrm{~cm})$} & \multirow{2}{*}{$t(\mathrm{~mm})$} & $\alpha_{c}$ & \multicolumn{2}{c}{ Load $(\mathrm{N})$} \\
\cline { 5 - 6 } & & & & Modeling & Experiment \\
\hline Experiment \# 12 & 12 & 8 & 0.45 & 1729 & 1863 \\
Experiment \# 2 & 11 & 4 & 0.40 & 1097 & 1457 \\
\hline
\end{tabular}

Table 2 compares the experimental results with the load at perforation predicted using the finite element model with parameters selected from red abalone. Considering the following simplifying assumptions made in the modeling, the results are in good agreement. In particular:

(1) The thickness of the seashell actually varies as a function of position on the shell. The thinnest part of the shell is usually observed at the rim of the shell while the thickest part appears in the middle. In the FEM model, a uniform shell thickness was considered through the whole structure.

(2) The actual seashell structure is asymmetric, whereas in this work, a spherical cap represented the shell.

(3) Porosity of calcite material influences the behavior of the shell. Nonetheless, a solid model of calcite free of defects was assumed.

\section{Concluding remarks}

Multiscale modeling and structural optimization have been used in this work to demonstrate that nacreous shells are optimized to resist perforation. The geometry of the shell at the macroscale and the micromechanics of the nacreous layer have been modeled, and three different failure modes were identified depending on the morphology of the shell. Parametric and optimization studies within the design space revealed that whenever two failure modes in different layers coincide, the shell shows optimum resistance to perforation by a sharp indenter. In this optimum configuration, the shell structure exploits the capabilities of the different layers of the shell at their full extent. Multiscale optimization of the seashell revealed a convergence of all parameters to a narrow range within the design space rather than converging to a single point. From the experiments performed on the two shells of red abalone, we concluded that the actual seashell has a microstructure design that fully exploits its material properties. The crack propagated through the thickness of the shell in three different failure modes. In addition, composed of typical ceramic material, the seashell could support up to $1900 \mathrm{~N}$ when loaded via a sharp indenter, which is surprisingly high compared to its size and structure.

From the multiscale optimization of the seashell structure and the experiments performed on actual shells of red abalone, some design guidelines for the synthesis of two-layer shells for protection purposes can be proposed and summarized as:

(1) The combination of a hard outer layer with a tough and ductile inner layer can lead to an ideal two-layer shell structure for protection purposes, such as a helmet.

(2) At the optimum solutions, we found that several failure modes occur simultaneously. In this case, the shell employs strategically its materials to postpone the catastrophic failure. Consequently, the thicker shells are not necessary stronger; rather, the shell can resist the highest load when a suitable combination of multiscale parameters is selected.

(3) At the microstructural level, a decrease in the thickness of tablet and interface of nacre with respect to the tablet length makes the material stiffer and stronger; however, consideration of a lower bound for those dimensions is necessary to avoid the tablet rupture.

(4) If the shell is supposed to be optimized against perforation with respect to both the geometry and the material, no unique solution exists, but the optimum solutions fall into a small region of the design space. In all case, however, local optima lie at points where multiple failure modes occur simultaneously.

\section{Acknowledgments}

This work was supported by the Faculty of Engineering of McGill University and by the Natural Sciences and Engineering Research Council of Canada, discovery grant program. We would like to thank Dr. Reza Rabiei for obtaining the SEM image of the fracture surface of nacre.

\section{References}

[1] Barthelat F. Biomimetics for next generation materials. Philosophical Transactions of the Royal Society A: Mathematical Physical and Engineering Sciences, 2007, 365, 2907-2919.

[2] Barthelat F, Tang H, Zavattieri P D, Li C M, Espinosa H D. On the mechanics of mother-of-pearl: A key feature in the 
material hierarchical structure. Journal of the Mechanics and Physics of Solids, 2007, 55, 306-337.

[3] Meyers M A, Lin A Y M, Chen P Y, Muyco J. Mechanical strength of abalone nacre: Role of the soft organic layer. Journal of the Mechanical Behavior of Biomedical Materials, 2008, 1, 76-85.

[4] Sarikaya M, Aksay I A. Biomimetics: Design and Processing of Materials (AIP Series in Polymers and Complex Materials), American Institute of Physics, New York, USA, 1995.

[5] Wang C H, Zhang L C, Mai Y W. Deformation and fracture of macadamia nuts. I. Deformation analysis of nut-in-shell. International Journal of Fracture, 1994, 69, 51-65.

[6] Fischer-Cripps A C. Introduction to Contact Mechanics, 2nd ed., Springer, New York, USA, 2007.

[7] Caspi E N, Pokroy B, Lee P L, Quintana J P, Zolotoyabko E. On the structure of aragonite. Acta Crystallographica Section B: Structural Science, 2005, 61, 129-132.

[8] Rabiei R, Bekah S, Barthelat F. Failure mode transition in nacre and bone-like materials. Acta Biomaterialia, 2010, 6, 4081-4089.

[9] Kotha S P, Li Y, Guzelsu N. Micromechanical model of nacre tested in tension. Journal of Materials Science, 2001, 36, 2001-2007.

[10] Wang R Z, Suo Z, Evans A G, Yao N, Aksay I A. Deformation mechanisms in nacre. Journal of Materials Research, 2001, 16, 2485-2493.

[11] Currey J D. Mechanical properties of mother of pearl in tension. Proceedings of the Royal Society of London Series B-Biological Sciences, 1977, 196, 443-463.

[12] Jager I, Fratzl P. Mineralized collagen fibrils: A mechanical model with a staggered arrangement of mineral particles. Biophysical Journal, 2000, 79, 1737-1746.

[13] Aboudi J. Mechanics of Composite Materials: A Unified Micromechanical Approach, Elsevier, New York, USA,
1991.

[14] Jackson A P, Vincent J F V, Turner R M. The mechanical design of nacre. Proceedings of the Royal Society of London Series B-Biological Sciences, 1988, 234, 415-440.

[15] Barthelat F, Li C M, Comi C, Espinosa H D. Mechanical properties of nacre constituents and their impact on mechanical performance. Journal of Materials Research, 2006, 21, 1977-1986.

[16] Barthelat F, Espinosa H D. An experimental investigation of deformation and fracture of nacre-mother of pearl. Experimental Mechanics, 2007, 47, 311-324.

[17] Courtney T H. Mechanical Behavior of Materials, McGraw-Hill, New York, USA, 1990.

[18] Fitoussi J, Guo G, Baptiste D. Determination of a tridimensional failure criterion at the fibre/matrix interface of an organic-matrix/discontinuous-reinforcement composite. Composites Science and Technology, 1996, 56, 755-760.

[19] Stamblewski C, Sankar B V, Zenkert D. Analysis of three-dimensional quadratic failure criteria for thick composites using the direct micromechanics method. Journal of Composite Materials, 2008, 42, 635-654.

[20] Menig R, Meyers M H, Meyers M A, Vecchio K S. Quasi-static and dynamic mechanical response of Haliotis rufescens (abalone) shells. Acta Materialia, 2000, 48, 2383-2398.

[21] Lawn B, Bhowmick S, Bush M B, Qasim T, Rekow E D, Zhang Y. Failure modes in ceramic-based layer structures: A basis for materials design of dental crowns. Journal of the American Ceramic Society, 2007, 90, 1671-1683.

[22] Nelder J A, Mead R. A simplex-method for function minimization. Computer Journal, 1965, 7, 308-313.

[23] Weaver P M, Ashby M F. Material limits for shape efficiency. Progress in Materials Science, 1997, 41, 61-128. 